\title{
"Double hit" B-lymphoblastic lymphoma with concurrent IGH/BCL2 and 8q24/MYC translocations: a case report
}

Lu He

Nanjing Drum Tower Hospital

Zhiwen Li

Nanjing Drum Tower Hospital

Xiangshan Fan ( $\sim$ fxs23@163.com )

Nanjing Drum Tower Hospital, the Affiliated Hospital of Nanjing University Medical School

Jieyu Chen

Nanjing Drum Tower Hospital

Hongyan Wu

Nanjing Drum Tower Hospital: Nanjing University Medical School Affiliated Nanjing Drum Tower Hospital

Yao Fu

Nanjing Drum Tower Hospital

Case Report

Keywords: double-hit, B-lymphoblastic lymphoma, 8q24/MYC, IGH/BCL2, case report

Posted Date: September 2nd, 2020

DOl: https://doi.org/10.21203/rs.3.rs-67816/v1

License: (c) (i) This work is licensed under a Creative Commons Attribution 4.0 International License.

Read Full License 


\section{Abstract}

\section{Background}

B-lymphoblastic lymphomas (B-LBLs) with a documented "double-hit" (DH) (IGH/BCL2 and 8q24/MYC rearrangement) are rare and their clinical, cytogenetic and immunophenotypic features have not been well elucidated. Here we describe an unusual case of de novo DH B-LBL.

\section{Case presentation}

A 39-year-old man presented with abdominal distention at admission. Radiological findings revealed a retroperitoneal lump and multiple lymphadenopathy. Histologic examination of the abdominal mass showed a predominant population of neoplastic lymphoblasts with round nuclei, which focally formed single files surrounded by fibrotic septa. These cells have high nuclear to cytoplasmic ratio, fine chromatin, and no inconspicuous nucleoli. Necrosis, apoptotic bodies and mitotic figures were common. Prominent starry-sky appearance was not observed. The typical immunophenotype of malignant cells was positive for TdT, CD99, CD10, PAX-5, Bcl2 (70\%), MYC (70\%), and negative for CD34,Cyclin-D1, SOX11, CD56, and CD123. The proliferation index was high with $\mathrm{Ki}-67$ of $80 \%$. Fluorescence in situ hybridization (FISH) studies demonstrated 8q24/MYC rearrangement and IGH/BCL2 gene fusion. Subsequent bone marrow biopsy showed no involvement. Normal chromosome karyotype was observed. Finally, the diagnosis of "double-hit" B-LBL was clinically rendered. The patient showed an initial response to six cycles of hyper-CVAD chemotherapy (hyper-fractionated cyclophosphamide, vincristine, doxorubicin, and dexamethasone) and was alive at the last follow-up (2020.4.10, six months after the diagnosis).

Conclusions

Few guidelines regarding DH B-LBL are available in the literature or in the WHO scheme. The optimal clinical management of such patients has not been fully established attributed to the small number of reported cases. Multicenter data is warranted to better make clinical strategies on patients with DH B-LBL.

\section{Background}

The term "double-hit" lymphoma (DHL) was introduced to describe a small group of aggressive lymphomas with co-occurrence of two oncogenic translocations, such as MYC, BCL2, BCL6, and CCND1 [1]. The most common DHLs that harbor a MYC rearrangement at chromosome 8q24 and a rearrangement in chromosome 18q21 account for an estimated $2 \%$ of all B-cell malignancies and have a heterogeneous histology with a dismal outcome [2]. Morphologically, these cases typically either resemble "B-cell lymphoma, unclassifiable, with features intermediate between diffuse large B-cell lymphoma (DLBCL) and Burkitt lymphoma (BL)" (most frequently), DLBCL, or less commonly bear a blastoid appearance [3]. Among them, B-lymphoblastic lymphomas (B-LBLs) with a documented "double- 
hit" (DH) (IGH/BCL2 and 8q24/MYC rearrangement) are rare and their clinical, cytogenetic and immunophenotypic features have not been well elucidated. Here we report such a case of DHB-LBL.

\section{Case Presentation}

A 39-year-old man complained of abdominal distention at admission in our hospital. Nothing special was found in the physical exam, and the patient denied of any past medical history or family history. A fullbody CT scan revealed multiple lesions of retroperitoneal soft tissue density wrapping surrounding vessels and ureter. Dilation of left renal pelvis and upper ureter, hydronephrosis and hydroureter indicated malignancy. Pelvic effusion was notable with multiple adenopathy in enterocoelia (max size: $3.3 \mathrm{~cm} * 1.2 \mathrm{~cm}$ ) and thickened peritoneum, which suggested the possibility of metastasis. PET/CT revealed mildly increased glucose uptake in most lymph node territories (SUV max $=3.5$ ), suggesting multiple involvement by lymphoma. Lactate dehydrogenase (LDH) was modestly elevated $(300 \mathrm{U} / \mathrm{L})$. Blood routine did not display any anomaly with hemoglobin $141 \mathrm{~g} / \mathrm{L}$, platelets $295 \times 10^{9} / \mathrm{L}$, WBC $6.3 \times$ $10^{9} / \mathrm{L}$. A core needle biopsy of the retroperitoneal soft tissue was then performed. Histologic examination yielded a diffuse infiltration of lymphoblasts varying from small-size to intermediate-size with round nuclei, which focally formed single files surrounded by fibrotic septa. These cells have high nuclear to cytoplasmic ratio, fine chromatin, and no inconspicuous nucleoli. Necrosis and apoptotic bodies were common. Mitotic figures were easily found. Prominent starry-sky appearance was not observed (Fig. 1a).

Immunophenotypic data regarding the cells of interest were derived from IHC with CD20-, PAX5+, CD79a+, CD10+, Bcl2+ (70\%), MYC+ (70\%), Bcl6-, MUM1-, CD30-, CD21-, Cyclin-D1-, SOX11-,CD56-, CD123-, TdT+, CD99+, MPO-, CD34-, CD117-, CD68 sparsely+, CD5( \pm ), CD3( \pm ); Ki-67 stained about $80 \%$ of the cells

(Fig. 1b).In situ hybridization for Epstein-Barr virus encoded RNA (EBER ISH) was negative. Subsequent bone marrow examination showed normal proliferation of hematopoietic cell without lymphoma involvement. Fluorescence in situ hybridization (FISH) performed on formalin-fixed paraffin-embedded (FFPE) mass tissues identified a breakpoint near the distal 5' end of MYC, and breakpoints within the IGH locus and proximal to BCL2 gene, respectively (Fig. 2). The patient bore normal chromosome karyotype. Immunoglobulin gene arrangement analysis was not available due to the limited amount of tissue. Accordingly, a diagnosis of DH B-LBL was established based on the morphology, immunophenotype, and FISH analysis. After receiving six cycles of Hyper-CVAD, the patient's symptoms alleviated and stayed alive at the last follow-up (2020.4.10, six months after the diagnosis).

\section{Discussion And Conclusion}

Lymphomas harboring concurrent IGH-BCL2/t(14;18)(q32;q21) and MYC/8q24 translocations, which are referred to as "double-hit" or "dual-hit" (DH) lymphomas, are a group of B-cell malignancies with a heterogeneous cytologic and histologic features. Rearrangements in MYC and BCL2 are extremely rare in B-LBL $[4,5]$. Such double-hit B-LBL cases may emerge de novo or through transformation of follicular lymphomas, both of which have been described to have an aggressive clinical course and poor prognosis $[2,6]$. 
In Subramaniyam et al.'s study, the patients with de novo DH B-LBL were adults with a median age of 59 years. They had complex chromosomal abnormalities, in addition to the BCL2 and MYC translocations. The reported median overall survival was 1.5 months (range, $<1-11$ months) [4]. Kelemen et al. also found the prognosis of B-LBL with a combined IGH/BCL2 and MYC translocation was poor, with a median survival of five months after the initial diagnosis (range,3-27 months) [7]. The present case was alive at the last follow-up (2020.4.10, six months after the diagnosis).

As to those TdT-positive neoplasms derived from a minority of follicular lymphomas (FL) and characterized by blastoid morphology, the current recommendation in the WHO-2016 scheme is to classify them as B-LBL transformed from follicular lymphoma, which is exceedingly rare as well, with fewer than 10 reported cases. Interestingly, most of these cases were featured with MYC rearrangement in addition to IGH/BCL2 fusion [2, 6, 8-13]. Lymphoblastic transformation of FL is thus proposed to have occurred due to a gain of MYC rearrangement at an early stage of FL lymphomagenesis (immature Bprecursor carrying $\mathrm{t}(14 ; 18)$ (q32;q21) in the bone marrow), and then the expression of $\mathrm{TdT}$ has been interpreted as either a "dedifferentiation" to a lymphoblastic (pre-B-cell) stage of B-cell development or as a re-expression of $\mathrm{TdT}$ in $\mathrm{B}$ cells due to accumulated somatic hypermutations, a hallmark of germinal center $B$ cells $[1,8-10,12,14,15]$.

Although TdT is critical in discriminating between TdT-positive LBL and TdT-negative aggressive B cell lymphomas, exceptional cases of either DH/TH-HGBCL with an aberrant expression of TdT have been reported, which could be quite a diagnostic pitfall. Morphology, immunophenotype and key genetic profiles may facilitate the differential diagnose $[8,16,17]$.

DH B-LBL should also be distinguished from entities like blastic plasmacytoid dendritic cell neoplasm (BPDCN), blastoid variant of mantle cell lymphoma (MCL), and myeloid neoplasm $[3,18,19]$. Among them, the reported BPDCNs have occurred mainly in elderly with a slight male predominance. The disease tends to involve multiple sites, most commonly the skin, followed by bone marrow and peripheral blood and lymph node. BPDCN is characterized by a diffuse, monomorphous infiltrate of medium-sized blast cells reminiscent of lymphoblasts or myeloblasts. The tumor cells express CD4, CD56, CD43, CD123 and lack expression of TdT, which distinct from LBL [3].Specific chromosomal aberrations are lacking, but complex karyotypes are common. Moreover, gene expression analysis of BPDCN reveals a unique signature, distinguished from those of myeloid and lymphoid acute leukemia [20].

As illustrated in the present case, strong expression of CD99 and TdT with lack of expression of CD20 in neoplastic cells has been used as features to support immaturity. Positive for PAX5 and CD79a demonstrated B-cell differentiation. The age of the patient, the location of the lesion, and tumor cells being negative for CD123 and CD56 were not supportive of BPDCNs. MCL-associated markers (CyclinD1, SOX11) were negative, and MPO was not detected, excluding acute myeloid leukemia or B/myeloid acute leukemia, and blastoid variant of mantle cell lymphoma, respectively. Finally, a diagnosis of DH B-LBL was established based on the morphology, immunophenotype, and FISH analysis. 
Of note, components of FL might be missed due to the limitations of biopsy. Theoretically, we cannot definitely rule out the possibility of lymphoblastic transformation of FL in this case. However, considering relatively low FDG uptake values and the patient denying oncologic history, there is insufficient evidence (either histologically or clinically) to support the setting of FL. De novo DH B-LBL was thus preferred.

At present, few guidelines regarding DH B-LBL are available in the literature or in the WHO scheme. The optimal clinical management of such patients has not been fully established attributed to the small number of reported cases. Multicenter data is warranted to better make clinicalstrategies on patients with DH B-LBL.

\section{Abbreviations}

DHL: double-hit lymphoma; DLBCL: diffuse large B-cell lymphoma; BL: Burkitt lymphoma; B-LBLs: Blymphoblastic lymphomas; EBER ISH: Epstein-Barr virus encoded RNA; FISH: Fluorescence in situ hybridization; FFPE: formalin-fixed paraffin-embedded; DH: double-hit or dual-hit; BPDCN: blastic plasmacytoid dendritic cell neoplasm; MCL: mantle cell lymphoma; FL: follicular lymphoma

\section{Declarations}

\section{Ethics approval and consent to participate}

This study was approved by the Pathology Department of Nanjing Drum Tower Hospital, China. Written informed consent was obtained from each subject.

\section{Consent for publication}

All authors consent to the publication of the manuscript in Diagnostic Pathology.

\section{Availability of data and materials}

The authors confirm that the data supporting the findings of this study are available within the article.

\section{Competing interests}

The authors have no competing interests to declare.

\section{Funding}

This study was supported by grants from Nanjing Medical Science and Technology Development Project (QRX17004 and ZKX17013).

\section{Authors' contributions}


Xiangshan Fan designed the experiments, Lu He and Zhiwen Li interpreted the results, and Lu He wrote the manuscript. Jieyu Chen, Hongyan Wu and Yao Fu performed the experiments. All authors read and approved the final manuscript

\section{Acknowledgments}

Not applicable.

\section{References}

1. Aukema SM, Siebert R, Schuuring E, et al. Double-hit B-cell lymphomas. Blood. 2011;117:2319-31.

2. Li S, Lin P, Fayad LE, et al. B-cell lymphomas with MYC/8q24 rearrangements and IGH@BCL2/t(14;18)(q32;q21): an aggressive disease with heterogeneous histology, germinal center B-cell immunophenotype and poor outcome. Mod Pathol. 2012;25:145-56.

3. Swerdlow S, Campo E, Harris NL, Jaffe ES, Pileri SA, Stein H, Thiele J. WHO classification of tumours of haematopoietic and lymphoid tissues. Revised 4th ed. Lyon: IARC;2017.

4. Subramaniyam S, Fraser CR, Rao PH, Feldman E, Ely S, Mathew S. De novo B lymphoblastic leukemia/lymphoma in an adult with $\mathrm{t}(14 ; 18)(\mathrm{q} 32 ; \mathrm{q} 21)$ and c-MYC gene rearrangement involving 10p13. Leuk Lymphoma. 2011;52:2195-9.

5. Liu W, Hu S, Konopleva M, et al. De Novo MYC and BCL2 Double-hit B-Cell Precursor Acute Lymphoblastic Leukemia (BCP-ALL) in Pediatric and Young Adult Patients Associated With Poor Prognosis. PediatrHematol Oncol. 2015;32:535-47.

6. Snuderl M, Kolman OK, Chen YB, et al. B-cell lymphomas with concurrent IGH-BCL2 and MYC rearrangements are aggressive neoplasms with clinical and pathologic features distinct from Burkitt lymphoma and diffuse large B-cell lymphoma. Am J Surg Pathol. 2010;34:327-40.

7. Kelemen K, Holden J, Johnson LJ, Davion S, Robetorye RS. Immunophenotypic and cytogenetic findings of B-lymphoblastic leukemia/lymphoma associated with combined IGH/BCL2 and MYC rearrangement. Cytometry B ClinCytom. 2017;92:310-4.

8. Geyer JT, Subramaniyam S, Jiang Y, et al. Lymphoblastic transformation of follicular lymphoma: a clinicopathologic and molecular analysis of 7 patients. Hum Pathol. 2015;46:260-71.

9. Ok CY, Medeiros LJ, Thakral B, et al. High-grade B-cell lymphomas with TdT expression: a diagnostic and classification dilemma. Mod Pathol. 2019;32:48-58.

10. De Jong D, Voetdijk BM, Beverstock GC, van Ommen GJ, Willemze R, Kluin PM. Activation of the Cmyc oncogene in a precursor-B-cell blast crisis of follicular lymphoma, presenting as composite lymphoma. N Engl J Med. 1988;318:1373-8.

11. Gauwerky CE, Haluska FG, Tsujimoto Y, Nowell PC, Croce CM. Evolution of B-cell malignancy: pre-Bcell leukemia resulting from MYC activation in a B-cell neoplasm with a rearranged BCL2 gene. Proc Natl Acad Sci U S A. 1988;85:8548-52. 
12. Kroft SH, Domiati-Saad R, Finn WG, et al. Precursor B-lymphoblastic transformation of grade I follicle center lymphoma. Am J ClinPathol. 2000;113:411-8.

13. Kaplan A, Samad A, Dolan MM, et al. Follicular lymphoma transformed to "double-hit" B lymphoblastic lymphoma presenting in the peritoneal fluid. Diagn Cytopathol. 2013;4:986-90.

14. Mamessier E, Broussais-Guillaumot $F$, Chetaille B, et al. Nature and importance of follicular lymphoma precursors. Haematologica. 2014;99:802-10.

15. Young KH, Xie Q, Zhou G, et al. Transformation of follicular lymphoma to precursor B-cell lymphoblastic lymphoma with c-myc gene rearrangement as a critical event. Am J ClinPathol. 2008;129:157-66.

16. Moench L, Sachs Z, Aasen G, Dolan M, Dayton V, Courville EL. Double- and triple-hit lymphomas can present with features suggestive of immaturity, including TdT expression, and create diagnostic challenges. Leuk Lymphoma. 2016;57:2626-35.

17. Slot LM, Hoogeboom R, Smit LA, et al. B-Lymphoblastic Lymphomas Evolving from Follicular Lymphomas Co-Express Surrogate Light Chains and Mutated Gamma Heavy Chains. Am J Pathol. 2016;186:3273-84.

18. Feldman AL, Arber DA, Pittaluga S, et al. Clonally related follicular lymphomas and histiocytic/dendritic cell sarcomas: evidence for transdifferentiation of the follicular lymphoma clone. Blood. 2008;111:5433-9.

19. Ratei R, Hummel M, Anagnostopoulos I, et al. Common clonal origin of an acute B-lymphoblastic leukemia and a Langerhans' cell sarcoma: evidence for hematopoietic plasticity. Haematologica. 2010;95:1461-6.

20. Sapienza MR, Fuligni F, Agostinelli C, et al. Molecular profiling of blasticplasmacytoid dendritic cell neoplasm reveals a unique pattern and suggests selective sensitivity to NF-kB pathway inhibition. Leukemia. 2014;28:1606-16.

\section{Figures}



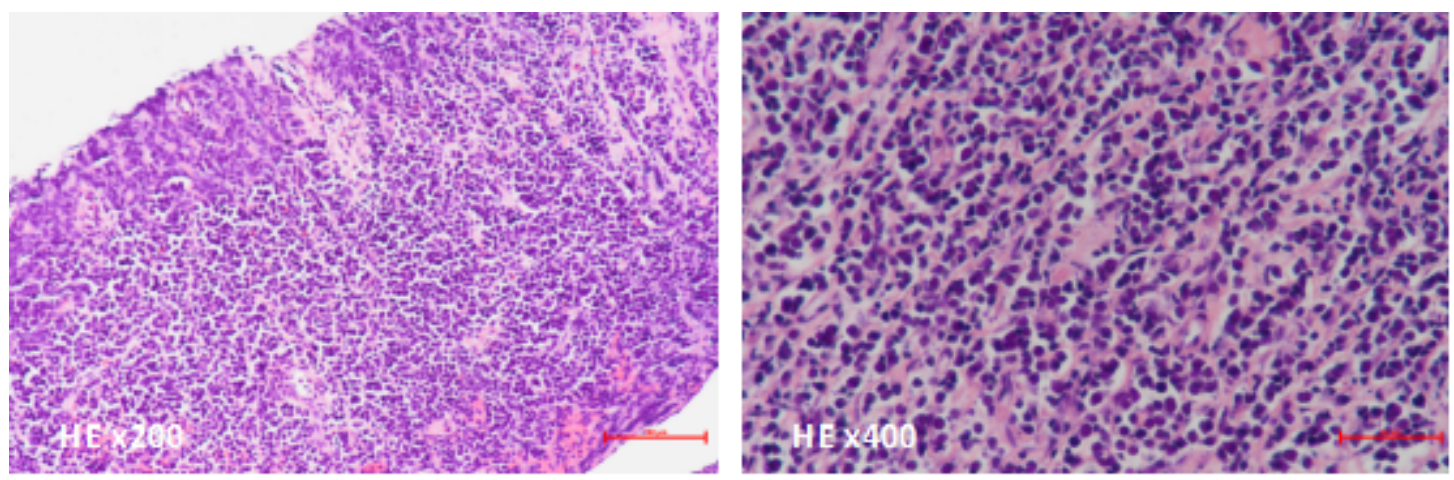

\section{A}
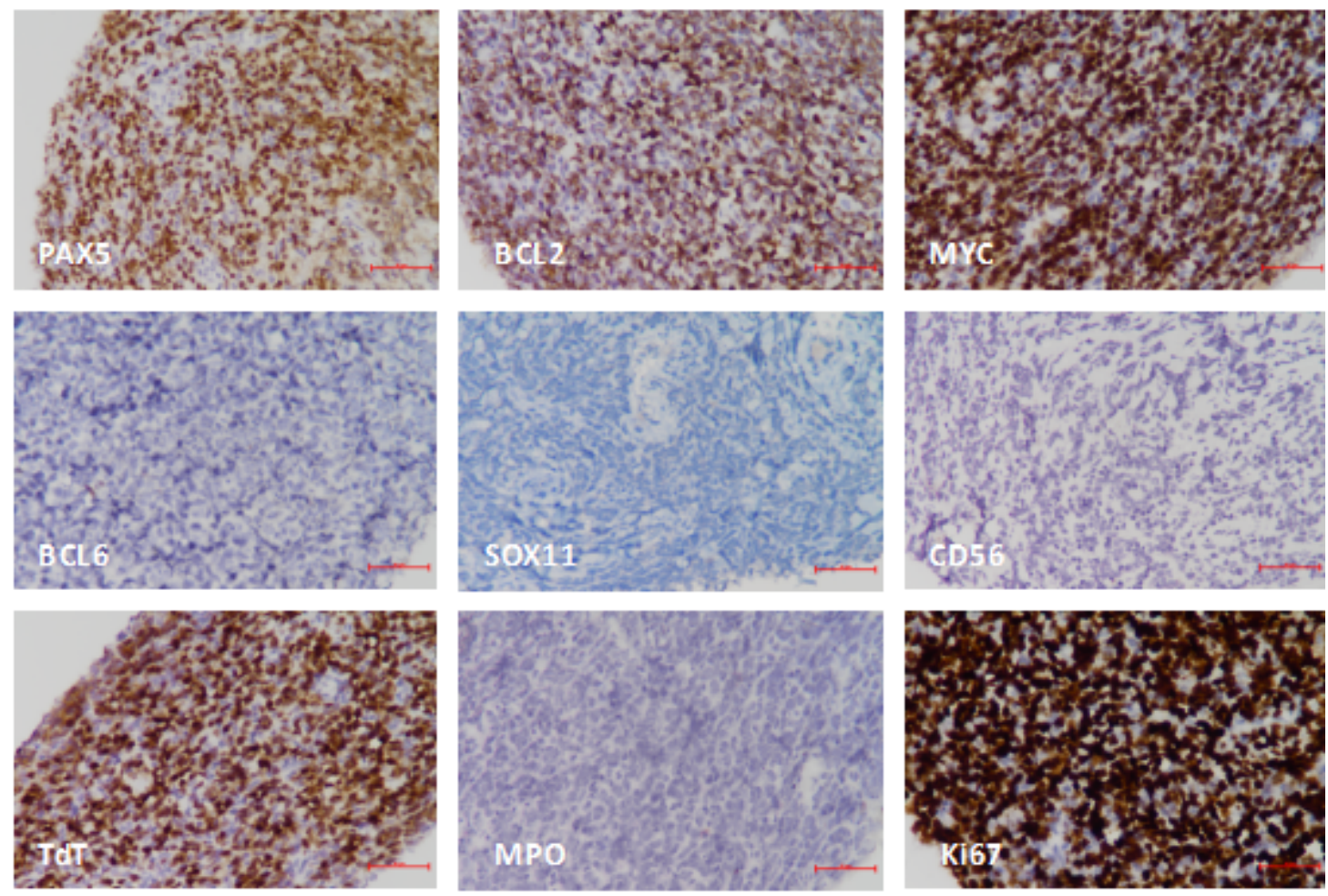

B

\section{Figure 1}

(a) Histological findings of the retroperitoneal lesion biopsy showed a diffuse, infiltration of small-sized to medium-sized lymphoblasts with round nuclei, high nuclear to cytoplasmic ratio, fine chromatin, and no inconspicuous nucleoli.(HE, x200 and x400, respectively) (b) The phenotype of the neoplastic cells was PAX5+, Bcl2+ (70\%), MYC+ (70\%), Bcl6-, SOX11-,CD56-, TdT+,MPO-; Ki-67 stained about $80 \%$ of the cells. $(x 400)$ 

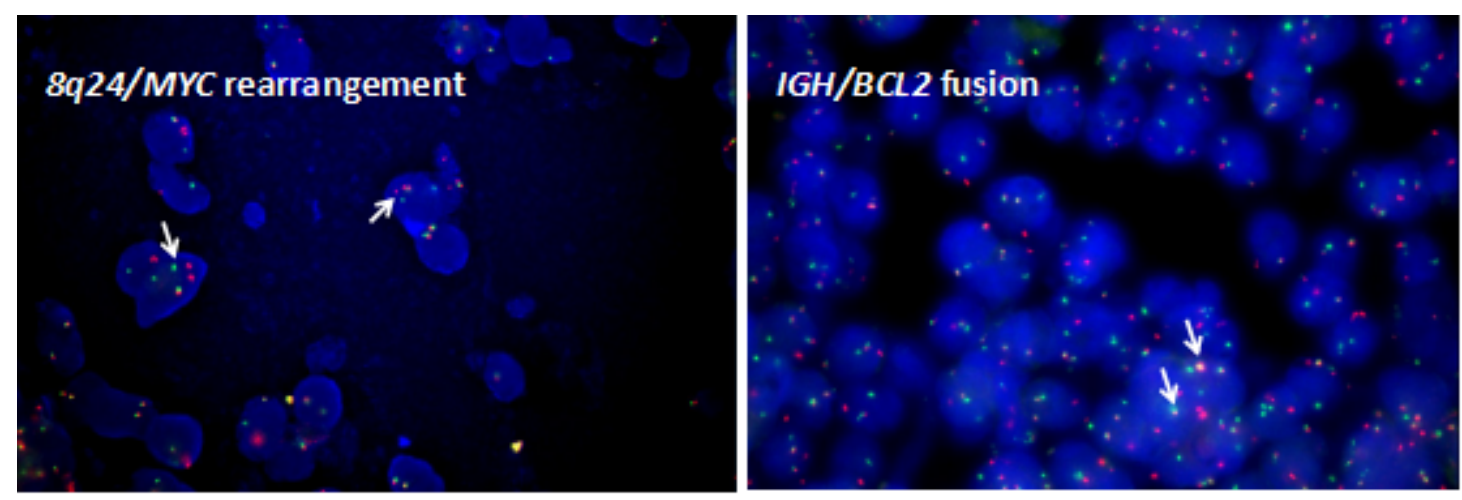

\section{Figure 2}

Interphase cells showed a signal pattern indicative of (a) 8q24/MYC rearrangement(break-apart probe) and (b) $t(14 ; 18)$ (q32;q21)/IGH-BCL2 (dual-color/dual-fusion probe) fusion by fluorescence in situ hybridization (FISH).

\section{Supplementary Files}

This is a list of supplementary files associated with this preprint. Click to download.

- CAREchecklist.pdf 